\title{
Diagnóstico por Doppler de la trombosis arterial causada por cateterismo umbilical
}

\author{
Juan Pablo Beca I. ${ }^{1}$; Janet Bloomfield G. ${ }^{2}$; Alberto Toso C. ${ }^{2}$; José M. Cortés E. ${ }^{2}$; \\ M. Luisa Glasinovic R. ${ }^{2}$; Ana María Marrese G. ${ }^{2}$
}

\author{
Diagnosis of neonatal femoral thrombosis following umbilical \\ arterial catheterization by ultrasonic flow detection
}

\begin{abstract}
At her second day of life, a 2,200 g, 34 weeks gestational age female newborn infant with idiopatic respiratory distress syndrome, under treatment with mechanical ventilation and monitored through an umbilical arterial catheter (UAC), had severe clinical signs of arterial obstruction of her left limb. Signs of oclussion at the cammon femoral artery level were evident at Doppler ultrasonography. Doppler determinations showed early arterial blood flow improvement before amelioration of skin coloration and arteriat pulses, that allowed close observation and withhoiding of surgical treatment which was finally not necessarv. Eightean newborn babies with an umbilical arterial catheter were then prospectively followed by Doppler ultrasound flow determinations. No fufther cases with clinical signs of arterial oclussion were found and Doppler studies performed in these late cases showed normal results in all of them. Doppler ultrasound flow determination is helptul for the diagnosis and management of lower limb arterial obstruction in newborn infants with umbilical arterial catheters.

(Key words: newborn, urnbifical artery catheterization. trombosis, femoral, diagnosis, Doppier, ultrasound.)
\end{abstract}

El empleo de la cateterización arterial umbilical en el cuidado intensivo de recién nacidos es habitual y de gran ayuda, pues permite obtener muestras de sangre evitando múltiples punciones, vigilar adecuadamente los gases de la sangre, medir la presión arterial e inyectar soluciones y medicamentos. Sin embargo, esta técnica no está exenta de complicaciones que incluyen infección, trombosis e hipertensión arterial de incidencia y frecuencia variables ${ }^{1-9}$.

En el diagnóstico y evaluación de las obstrucciones yasculares se han empleado diversos métodos ${ }^{1,8,10-14}$, entre ellos la ecografía, arteriografía con medio de contraste, cintigrafía y ultrasonografía Doppler. De esta última hay escasas publicaciones en recién nacidos $11,14,15$.

Después de un caso de obstrucción arterial demostrado y seguido con Doppler ultrasónico, se diseño un estudio para evaluar este método en recién nacidos. El propósito de esta publicación es describir el caso clínico que lo motivó y mostrar la validez de la ultrasonografja Dop-

1. Departamento de Pediatría. División Oriente: Universidad de Chile.

2. Clínica Alemana de Santiago. pler en lá observación y manejo de la obstruc. ción arterial de miembros inferiores en recién nacidos.

\section{Material y Método}

Se analizó el diagnóstico y la evolución de un recién nacido que sufrió obstrucción arterial de la extremidad inferior izquierda, asociada a cateterismo arterial umbjlical, confirmada y seguida su evolución mediante Doppler ultrasónico, a raíz del cual se diseñó un seguimiento prospectivo de 18 recién nacidos, atendidos en el servicio de neonatología de la Clinica Alemana de Santiago, entre octubre de 1988 y abril de 1989 , en los cuales fue necesario emplear un catéter arterial umbilical por indicación médica.

El estudio se rẹlizó en el servicio de recién nacidos, en la misma incubadora o cuna radiante del paciente, mediante uл equipo Medasonics con Doppler ultrasónico bjdireccional de $8 \mathrm{MHz}$ de frecuencia $y$ registro en papel; paralelamente se hicieron registros fotopletismográficos, con el propósito de obtener una expresión objetiva de la circulación capilar.

El peso de nacimiento promedio de los 18 casos estudiados fue $1.813 \mathrm{~g}$ (límites 960 y $4.710 \mathrm{~g}$ ) y gu edad gestacional promedio fue 31,6 semanas (límites 28 y 40). El diagnóstico de su problema de base fue mem. brana hialina en 15 niños, encefalopatía hipóxicoisquémica en 2 y atresia esofágica en uno. Todos estaban conectados a ventilador mecánico y se les había 
instalado un catéter arterial umbilical Argyle, calibres 3,5 ó $5 \mathrm{Fr}$, ubicado en posición alta, entre D4 y D11, comprobada radiológicamente en cada caso. Los catéteres se instalaron entre el primero y tercer día de vida, se mantuvieron por ? dias en promedio y se retiraron entre $\operatorname{los} 3$ y los 17 días de edad. A través de ellos se perfundieron soluciones de glucosa, de electrolitos $y$ y antibióticos, cuya naturaleza $y$ cantjad se registró detalladamente. En ningún caso se administró alimentación parenteral por la vía mencionada. En todos los pacientes se registraron los fenómenos vasomotores observados en las extremidades inferjores. E] estudio con Doppler $\infty$ hizo 24 horas y 6 días después de colocar cl catéter en todos los casos, repitiéndolo dos dias después de retirarlo en aquellos en quienes se mantuvo por más de 7 días. De acuerdo con el protocolo se haría ecografía arterial cuando se presentaran signos de obstrucción arterial.

\section{Resultados}

El caso inicial fue el de una niña recién nacida de 34 semanas de edad gestacional y $2.220 \mathrm{~g}$ de peso al nacer, con membrana hialina que requirió ventilación mecánica. Al segundo día de vida, después de colocar un catéter arterial umbilical, cambió el color de su extremidad inferior izquierda y desaparecieron los pulsos femoral y pedio, situación que persistió a pesar de haber retirado el instrumento. El examen de la extremidad can Doppler ultrasónico mostró significativa disminución del flujo de sangre en la artetia femural común izquierda, desaparición del mismo en los segmentos más distales y aplanamiento del registro fotopletismográfico (figura 1), ambos signos de obstrucción arterial Después de diez horas de observación, ante la eventualidad de que fuese necesario interrenirla quirúrgicamente para aliviar el obstáculo, a pesar de persistir sus manifestaciones clínicas, el Doppler mostró aumento del pulso femoral y aparición de flujo poplíteo. Con posterioridad el color de la extremidad afectada y los pulsos mejoraron progresivamente. Al cuarto dia se demostró con ultrasonido que había buen flujo de sangre hasta la arteria tibial posterior (figura 2) y a los 9 días hasta la pedia, mejorando concomitantemente la calidad del registro fotopletismográfico del pie. A los 30 djas, en un control ambulatorio, se demostió completa normalidad de los pulsos arteriales mediante Doppler y fotopletismografia.

En ringuno de los recién nacidos con catéter umbilical seguidos prospectivamente, se detec. taron fenómenos vasomotores de las extremidades inferiores, habiéndose completado 56 evaluaciones con Doppler ultrasónico y fotopletismo-

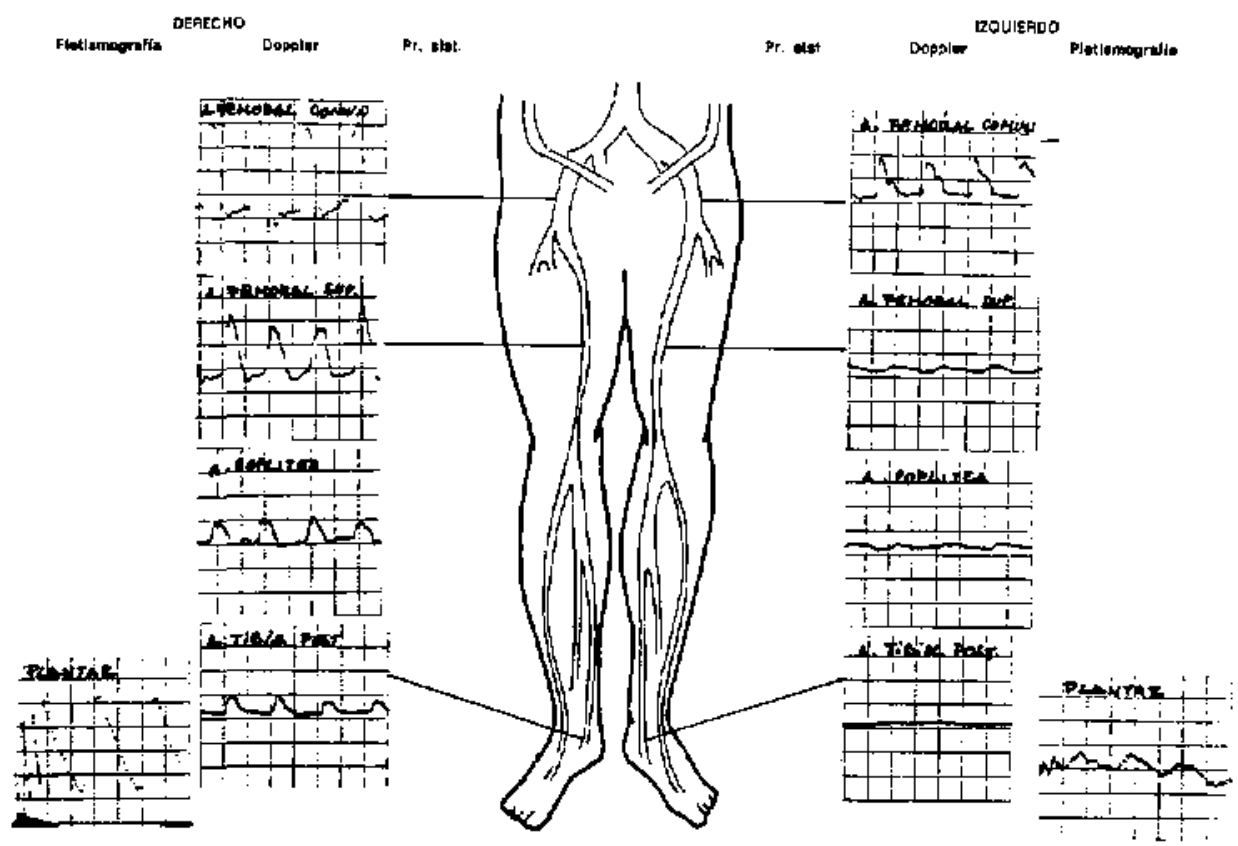

Fig. 1: Estudio arterial de miembros inferiores con técnica de ultrasonografia Doppler y fotopletismografía de los pies. Se aprecia disminución de flujo arterial en la arteria femoral común izquierda y desaparición hacia distal. 


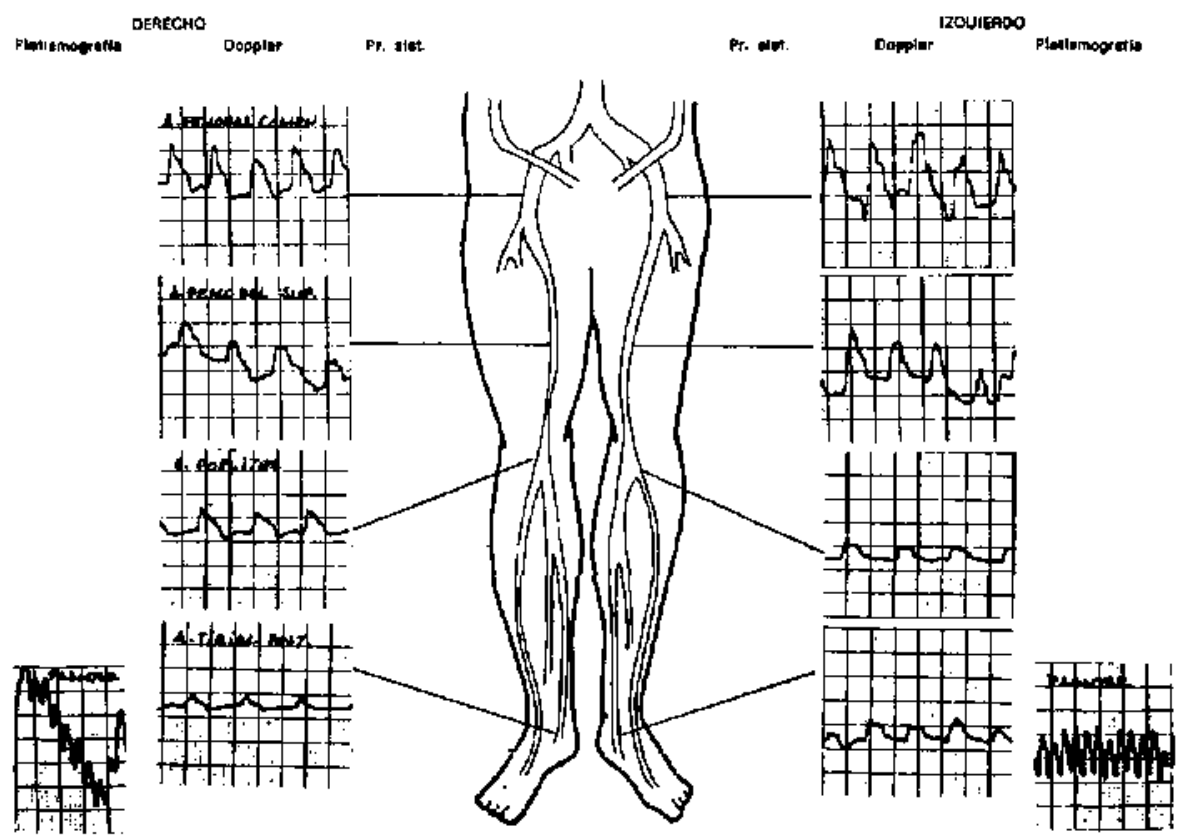

Fig. 2: Estudio arterial en el mismo paciente cuatro días después. Se aprecia reaparición de pulsos hasta la arteria pedia.

grafia. Todos sus resultados fueron concordantes con la ausencia clínica de signos de obstrucción vascular y mostraron indemnidad de flujo arterial de ambas extremidades desde la arteria femoral hasta la pedia. Las exploraciones fueron siempre fáciles de realizar y no se observaron complicaciones ni artefactos que dificultaran su interpretación.

\section{Discusión}

Las complicaciones trombóticas en las cateterizaciones arteriales umbilicales son relativamente frecuentes en la aorta y las arterias renales ${ }^{1-3}$. Una de sus expresiones clínicas más frecuentes es la hipertensión arterial ${ }^{6,9}$. La trombosis aórtica puede causar trombos femorales y lesiones isquémicas graves de miembros inferiores que pueden llegar hasta la amputación de ortejos o de una extremidad ${ }^{14}$. En el diagnóstico y control de este tipo de alteraciones vasculares se ha empleado el Doppler ultrasónico, que es útil para demostrar la obstrucción y cuantificar el flujo arterial11, 14, 15. Como tratamiento de las obstrucciones arteriales de extremidades se han propuesto la trombectomía quirúrgica ${ }^{16,13}$ y los agentes trombolíticos ${ }^{15,18,19}$, ambos con altos riesgos de fracaso y hemorragias, que esta técnica permite diferir indjcándoselos sólo cuando muestra evidencia de evolución desfavorable. En el caso presentado fue posible esperar y observar sin intentar tratamiento quirúrgico o trombolítico gracias a que se demostró reaparición del flujo sanguíneo a pesar de persistir el blanqueamiento total de la extremidad y la ausencia de pulsos, demostrando así la utilidad del método en el manejo de esta complicación de la cateterización arterial umbilical.

El estudio prospectivo demostró que la técnica es factible y de fácil interpretación. Como el Doppler ultrasónico es aplicable en ambos lados, es posible detectar, por comparación, pequeñas disminuciones de flujo y el nivel en que éstas se producen. El hecho de no haberse encontrado otros casos con obstrucción arterial hace suponer que ellas no son frecuentes en las extremidades inferiores, pero las características de esta experiencia no permiten obtener conclusiones sobre su incidencia. También debe tenerse presente que esta técnica no excluye la existencia de fenómenos trombóticos en la vecindad de las arterias renales.

En sintesis, la ultrasonografia Doppler en extremidades inferiores permite hacer el diagnós- 
tico y facilita el manejo de casos de obstrucción arterial en el período neonatal. Su uso está indicado cuando se sospecha clínicamente obstrucción arterial parcial o total, para confirmar el diagnóstico, seguir su evolución y definir el tratamiento con más precisión y menos riesgo.

\section{Resumen}

Una niña recién nacida de 34 semanas de edad gestacional con sindrome de dificultad respiratoria idiopática, en tratamiento con ventilador mecánico (IPPV), sufrió obstrucción de la arteria femoral común izquierda, asociada al empleo de un catéter umbilical. Esta fue demostrada con Doppler ultrasónico, mediante el cual fue también posible determinar precozmente la reaparición de flujo sanguíneo, a pesar de persistir la ausencia de pulsos y el blanqueamiento de la extremidad, lo que hizo innecesario el empleo de tratamiento quirúrgico o trombolítico. En otros 18 recién nacidos sometidos a cateterización arterial umbilical y estudiados prospectivamente mediante el método aludido y fotopletísmografía no se encontraron otros casos de obstrucción vascular de las extremidades inferiores, demostrándose la concordancia de resultados normales en los estudios mediante Doppler ultrasónico, con ausencia de signos clínicos del trastorno. La ultrasonografía Doppler permite confirmar el diagnóstico y facilita el mantejo de casos de la obstrucción arterial de extremidades inferiores que puede ocurrir en recién nacidos tratados con catéteres umbilicales.

(Palabras claye: recién nacido, catéter arterial umbilical, trombosis femoral, ultrasonido, Doppler ultrasónico.)

\section{Referencias}

1. Neal, W.A.; Reynolds, J.W.; Jarvis, C.W; Williams, H.J.: Umbilical artery catheterization: Demonstration of arterial thrombosis by aortography. Pe. djatrics $1972 ; 50: 6-13$.

2. Wigger, H.J.; Bransitver, B.R.: Blane, W.A.: Thromboses due to catheterization in infants and children. J. Pediatr 1970; 76: 1-11.

3. Goetzman, B.W.; Stadalnik, R.C; Bogren, H.G. Blonkeship, W.J.; Ikeda, R.M.; Thayer, J.: Thrombotic complications of umbilical artery catheters: a clinical and radiologis study. Pediatrics 1975: $56: 374-9$.

4. Topia, J.L.; Ventura, P.; González, H.; Winter, A.: Juez, $G_{*}$ : Cateterismo Umbilical Arterial. Seis años de experiencia. Rey Chil Pediatr 1986 57: 133-137.
5. Du, J.N.H.; Briggs, J.N.; Young, G.: Disseminated intravascular coagulopathy and hyaline membrane disease: massive thrombosis following umbilicat astery catheterization. Pediatrics $1970 ; 45: 287-290$.

6. Buer, S.T.; Feldman, SM.: Gellis, S.S.; Retik, A,B.: Neonatal Hypertension. A complication of umbilical catheterization. N Engl J Med 1975; 293: 1032-3.

7. Coplan, M.S.; Cohn, R.A.: Langman, C.B.; Con way, J.A.; Shkolnik, A.; Brouillette, R.T: Favorable outcome of neonatal aortic thrombosis and renovascular hypertension. J Pediatr 1989; 115: $291-5$.

8. Vailas, G.N.; Brouillette, R.T.; Scott, J.P.; Shkol. nik, A.; Conway, $J_{\text {; }}$ Wiringa. $K$.; Neonatal aortic thrombosis: Recent experience. J Pediatr 1986; 109: 101-8,

9. Notero, C.; Oto, M.A ; Morales, B.; Benavities, A.; Fuentealba, S.; Lagos, E.; Amaral, H.; Martinez, $V$. . Trombosis de arteria renal en recién nacidos sometidos a cateterización de la arteria umbilical. Rev Chil Pediatr 1989; 60: 346-352.

10. Giargiana, FA.: Siegel, M.E.; James, $A E_{\text {; }}$ Rho. des, B.A.: Wagner, H.M ; White, R.I.: A preliminary report of the complementary roles of arteriography and perfusion scanning in assessment of peripheral vascular disesse. Radiology 1973;108: $619-627$.

11. Strondness, D.E.; Schultz, R.D.; Summer, D.S.; Rushmer, R.F.: Ultrasonic flow detection. A useful technic in the evaluation in peripheral vascular disease. Am J Surg 1967; 113: 311-320.

12. Houston, A.B.; Garg, A.K, Maclaurin, J.C; Mackenzie, J.R.: Ultrasound positioning of umbilical arterial catheters. Lancet $1982 ; 2: 759-760$.

13. Oppenheimer. D.A.; Carroll, B.A.; Garth, K.E.: Ultrasonic detection of complications following umbilical arterial catheterization in the neonate. Radiology 1982;145: 667-672.

14. Puppala, B.L.; Benawara, R.; Mangurten, H.H.; Naidu, $S_{\text {.: }}$ Shirazi, $P_{\text {.: }}$ Doppler flow and radionuclide scan studies in the evaluation and monagement of peripheral axtery thrombosis in the neonate. J Pediatr 1981; $99: 791-4$.

15. Kenney, L.A.; Drummond, W.H.; Knight, M.E.: Millsaps, M.M.; Willioms, J.L.: Succesful treatment of neonatal aortic thrombosis with tissue plasminogen activator. J Pediatt 1990; 116: 798-801.

16. Payne, R.M.; Martin, T.C.; Bower, R.J.; Canter, C.E.: Management and follow-up of arterial thrombosis in the neonatal periox. J Pediati $1989 ; 114$ : 853-8.

17. Martin, J.E.; Moren, J.F,; Cook, L.S.; Goertz, $X . K$. .; Mottioli, L.: Neonatal aortic thrombosis complicating umbilical artery catheterization: Successful treatment with retroperitoneal aortic thrombectomy. Surgery 1989;105:793-6.

18. Emami, A.: Saldanha, R.; Kntpp, C.; Kodroff, $M .:$ Failure of systemic thrombolytic and heparin therapy in the treatment of neonatal aartjc throm. bosis. Pediatrics $1987 ; 79: 773-7$.

19. Strife, A.L.; Ball, W.S.; Towbin, R.; Keller, M.S.; Dillan, T.t Arterial oclusions in meonates: Use of fibrinolytic therapy. Radiology $1988 ; 166$ : 395 . 400 . 